\title{
Direccionamiento estratégico: Un Análisis en el contexto de las empresas de fluidos de perforación
}

\author{
Strategic direction: An analysis in the context of drilling fluid companies \\ Direção estratégica: Uma análise no contexto das empresas de fluidos de \\ perfuração
}

Recibido: mayo 2019

Arbitrado: junio 2019

Publicado: septiembre 2019
《 Shera Camejo

shera.camejof@gmail.com

ORCID: 0000-0002-1461-8325

Universidad del Zulia, Núcleo Costa Oriental del Lago, Venezuela
RESUMEN

La dirección estratégica permite alinear a toda la empresa en el mismo derrotero y fortalecer aquellos comportamientos que contribuyen al logro de los objetivos planteados, la investigación tuvo como objetivo, analizar el direccionamiento estratégico en las empresas de fluido de perforación. Utilizando el enfoque descriptivo, con diseño de campo, no experimental, transeccional, se aplicó una encuesta a los gerentes y coordinadores estratégicos de las cinco (5) empresas de fluidos de perforación ubicadas en la división Sur del Lago Trujillo. Los datos arrojados indican que es importante orientarse a realizar una mejor gestión y planificación estratégica dentro de la organización. En particular, en las empresas bajo estudio el direccionamiento estratégico representa un enfoque gerencial que permite a la alta dirección determinar un rumbo claro, y promover las actividades necesarias para que toda la organización trabaje en la misma dirección.

Palabras clave: Dirección; direccionamiento estratégico; gestión; organización, planificación estratégica
The strategic direction allows the entire company to be aligned on the same path and to strengthen those behaviors that, in order to achieve the objectives, the objective of the research was to analyze the strategic direction in drilling fluid companies. Using the descriptive approach, with field design, non-experimental, transectional, a survey was applied to the managers and strategic coordinators of the five (5) drilling fluid companies located in the South division of Lake Trujillo. The data obtained indicate that it is important to focus on better management and strategic planning within the organization. In particular, in the companies under study, strategic direction represents a managerial approach that allows senior management to determine a clear course, and promote the activities necessary for the entire organization to work in the same direction.

Keywords: Direction; strategic direction; management; organization, strategic planning
RESUMO
O direcionamento estratégico permite que toda a empresa esteja alinhada no mesmo caminho e fortalecer aqueles comportamentos que, para atingir os objetivos, o objetivo da pesquisa foi analisar o direcionamento estratégico em empresas de fluidos de perfuração. Utilizando a abordagem descritiva, com desenho de campo, não experimental transversal, foi aplicada uma survey aos gestores e coordenadores estratégicos das cinco (5) empresas de fluidos de perfuração localizadas na divisão sul do Lago Trujillo. Os dados obtidos indicam que é importante focar na melhor gestão e planejamento estratégico da organização. Em particular, nas empresas em estudo, a direção estratégica representa uma abordagem gerencial que permite à alta administração definir um rumo claro e promover as atividades necessárias para que toda a organização trabalhe na mesma direção.

Palavras-chave: Endereço; direção estratégica; gestão; organização, planejamento estratégico 


\section{INTRODUCCIÓN}

$\mathrm{T}$ odas las empresas a nivel mundial tienen una meta en común: lograr sus objetivos con el mayor de los éxitos; por lo cual, los gerentes y altos directivos de dichas organizaciones, plantean la creación de un plan que permita organizar de forma estratégica cada una de las acciones a llevar a cabo, obteniendo así el cumplimiento efectivo de cada uno de sus propósitos.

Para lograr esto, las empresas hacen uso de la dirección estratégica, vista como el conjunto de análisis, decisiones y acciones que una organización lleva a cabo para crear y mantener ventajas competitivas (Dess y Lumpkin, 2003) y una herramienta administrativa de la alta dirección de las empresas, cuyo resultado se refleja en el plan estratégico (Huisa, 2007). Mediante su aplicación, la organización decide sobre los recursos que serán utilizados y las políticas que requieren para la consecución de dichos objetivos (Rojas y Medina, 2012).

De esta forma, el proceso gerencial, permiten alinear las metas y los recursos de la organización con las oportunidades cambiantes de mercadeo. De manera que, la planeación estratégica es el proceso por el cual una empresa desarrolla los objetivos y las acciones concretas para llegar al estado deseado.

Atendiendo a estas consideraciones, queda claro que en la actualidad todas las organizaciones, ya sean públicas o privadas, de pequeño, mediano o gran tamaño cuentan con planes estratégicos que les permitan orientarse a la consecución de sus objetivos y metas con la finalidad de que se optimicen recursos y se dé cumplimiento a lo establecido, ya sea en los planes operativos o en los objetivos planteados.

Tal es el caso de las empresas del ramo petrolero concretamente en los fluidos de perforación, las cuales trabajan con la planificación estratégica como una nueva manera de ver las cosas, y ha hecho evidente, el enorme potencial que representa un sistema bien diseñado para avanzar en el cumplimiento de las metas estratégicas de la empresa.

Bajo este contexto, el objetivo central de la investigación fue analizar el direccionamiento estratégico en las empresas de Fluido de Perforación, con ello se busca darles a los gerentes de estas empresas los elementos necesarios para que estén preparados y puedan enfrentar los cambios del entorno, así como las situaciones complejas y no rutinarias que la actividad gerencial requiere.

\section{MÉTODO}

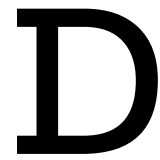
e acuerdo a la problemática planteada referida al direccionamiento estratégico en las empresas de Fluido de Perforación, el presente estudio se clasificó como descriptivo, el cual tiene como finalidad describir, analizar, e interpretar sistemáticamente características de los fenómenos estudiados sobre la realidad. 
En este sentido Hernández, Fernández y Baptista (2010), definen los estudios descriptivos como aquellos que buscan especificar las propiedades importantes de personas, grupos, o cualquier otro fenómeno sometido a un análisis. A su vez, el estudio presentó un diseño de campo, no experimental, transeccional, refiriéndose a la ubicación de la investigadora en el lugar de los hechos, logrando una obtención y manejo confiable de los datos, tomándolos en forma directa de la realidad donde se presentan en un momento único durante el proceso de investigación.

La técnica utilizada en el estudio fue la encuesta, mediante el cuestionario. El instrumento estuvo estructurado por 18 ítems, diseñado con un escalamiento de cinco alternativas de respuestas, aplicado a 5 gerentes y 5 coordinadores estratégicos. El instrumento diseñado fue sometido a un proceso de validación de contenido, a través de la técnica del "Juicio de Expertos" y para su confiabilidad se procedió a utilizar el coeficiente de Alpha Cronbach, mediante la utilización del paquete estadístico para las Ciencias Sociales (SPSS), versión 21,0 a través de la ruta del análisis de la fiabilidad, resultando 0,92.

\section{RESULTADOS}

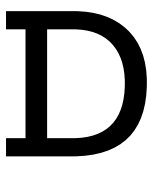

e acuerdo a los resultados mostrados en la tabla 1 referidos a los principios corporativos, en el el ítem 1 se evidencia frecuencias de $80 \%$ y $20 \%$ agrupadas en las opciones siempre y casi siempre, indicando tendencia positiva, al considerar los sujetos encuestados que dentro de la empresa en la que laboran los principios corporativos son considerados de gran importancia. Adicional a esto, se evidencia una media de 4,80 ubicándose en la categoría muy alta aplicación con respecto al baremo de interpretación de la misma.

Tabla 1. Principios corporativos.

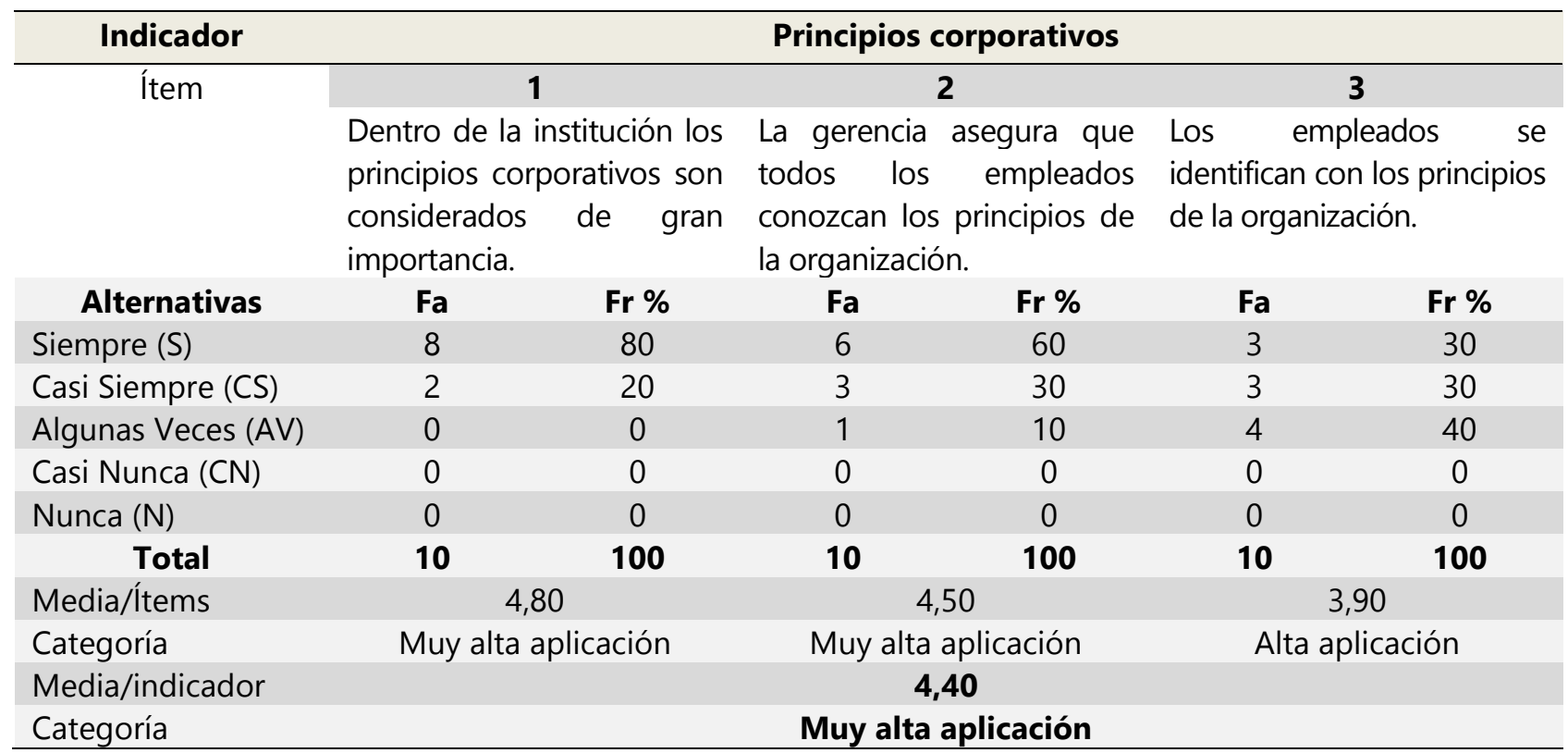


Asimismo, para el ítem 2 los resultados correspondientes señalan frecuencias de $60 \%$ y $30 \%$ agrupadas en las opciones siempre y casi siempre, con tendencia positiva, afirmando los encuestados que la gerencia asegura que todos los empleados conozcan los principios de la organización. Al mismo tiempo le acompaña una media de 4,50 colocándose en la categoría muy alta aplicación en referencia al baremo de interpretación.

De igual forma, el ítem 3 obtuvo frecuencias de 30\% y 30\% agrupadas en las opciones siempre y casi siempre, con tendencia neutral, afirmando los encuestados que los empleados se identifican con los principios de la organización. Adicionalmente, su media se posiciono en 3,90 ubicándola en la categoría de alta aplicación.

Así pues, se observa un promedio de 4,40 indicando muy alta aplicación según las respuestas dadas por los encuestados, poniendo en evidencia que dentro de las empresas de fluidos de perforación ubicadas en la división Sur del Lago Trujillo, los principios corporativos coinciden con lo expuesto por Serna (2008), para quien los mismos definen aspectos importantes para la organización y que deben ser compartidos por todos, constituyendo la norma de vida corporativa y el soporte de la cultura organizacional.

Con los valores alcanzados para el indicador, se permitió considerar que los principios corporativos son parte fundamental del direccionamiento estratégica, por lo tanto, estos deben ser evaluados para establecer si es necesario definirlos o redefinirlos al ser el punto de partida para dicho proceso. Por tanto, desde este contexto el direccionamiento estratégico induce a que la organización construya sus normas de vida corporativa a fin de que sean el soporte de su cultura organizacional.

Con respecto a lo presentado en la tabla 2, con relación al indicador misión, se observa que para el ítem 4 se muestran frecuencias de 10\% y 70\% correspondientes a las opciones siempre y casi siempre, con tendencia positiva, afirmando los encuestados que el principal propósito de la organización se encuentra formulado dentro de la misión. Por su parte, se obtuvo una media de 3,90 la cual se ubicó en la categoría de alta aplicación dentro del baremo.

En lo que se refiere al ítem 5, se evidencia frecuencias de $10 \%$ y $20 \%$ agrupadas en las opciones siempre y casi siempre, señalando una tendencia negativa afirmando que los empleados algunas veces se interesan en conocer la misión de la organización. Al mismo tiempo le acompaña una media de 3,40 situándose en la categoría alta aplicación. 
Tabla 2. Misión.

\begin{tabular}{|c|c|c|c|c|}
\hline Indicador & \multicolumn{4}{|c|}{ Misión } \\
\hline \multirow[t]{2}{*}{ Ítem } & \multicolumn{2}{|c|}{4} & \multicolumn{2}{|c|}{5} \\
\hline & \multicolumn{2}{|c|}{$\begin{array}{l}\text { El principal proposito de } \\
\text { organización es formulado dentro d } \\
\text { la misión de la misma. }\end{array}$} & \multicolumn{2}{|c|}{$\begin{array}{l}\text { Los empleados se interesan en } \\
\text { conocer la misión de la organización }\end{array}$} \\
\hline Alternativas & $\mathbf{F a}$ & Fr \% & $\mathbf{F a}$ & Fr \% \\
\hline Siempre (S) & 1 & 10 & 1 & 10 \\
\hline Casi Siempre (CS) & 7 & 70 & 2 & 20 \\
\hline Algunas Veces (AV) & 2 & 20 & 7 & 70 \\
\hline Casi Nunca (CN) & 0 & 0 & 0 & 0 \\
\hline Nunca (N) & 0 & 0 & 0 & 0 \\
\hline Total & 10 & 100 & 10 & 100 \\
\hline Media/Ítems & \multicolumn{2}{|c|}{3,90} & \multicolumn{2}{|c|}{3,40} \\
\hline Categoría & \multicolumn{2}{|c|}{ Alta aplicación } & \multicolumn{2}{|c|}{ Alta aplicación } \\
\hline Media/indicador & \multicolumn{4}{|c|}{3,65} \\
\hline Categoría & \multicolumn{4}{|c|}{ Alta aplicación } \\
\hline
\end{tabular}

Por otra parte, se observa un promedio de 3,65 que demuestra una alta aplicación de acuerdo a lo señalado por los encuestados, haciendo notar que las empresas objeto de estudio concuerdan con David (2013), quien puntualiza que la misión afirma el propósito que distingue una empresa entre otras similares.

Dichos resultados obtenidos para el indicador, permitieron considerar que, la misión como la razón de ser de la empresa, determinando las funciones a desempeñar en un entorno determinado, siendo la misma estable a lo largo del tiempo. En términos globales, dentro del direccionamiento estratégico, la misión incluye un plano general del nivel de los objetivos y finalidades que no deben ser confundidos con la visión al expresar el quehacer de la empresa de los planes y proyectos que entrega a terceros, y de la calidad con que se compromete a realizarlos.

La tabla 3 muestra los resultados para el indicador visión, iniciando con el ítem 6 donde se evidencian frecuencias de $50 \%$ y $10 \%$ para las opciones siempre y casi siempre, presentando una tendencia neutral que certifica que dentro de la visión siempre se ve reflejado lo que la empresa desea lograr en el futuro, mostrándose también una media de 4, colocándose en la categoría alta aplicación con respecto al baremo de interpretación de la misma. 
Tabla 3. Visión.

\begin{tabular}{|c|c|c|c|c|c|c|}
\hline \multirow{3}{*}{$\begin{array}{c}\text { Indicador } \\
\text { Ítem }\end{array}$} & \multicolumn{6}{|c|}{ Principios corporativos } \\
\hline & \multirow{2}{*}{\multicolumn{2}{|c|}{$\begin{array}{l}6 \\
\text { Lo que se desea lograr en } \\
\text { el futuro se ve reflejado } \\
\text { en la visión de la empresa }\end{array}$}} & \multicolumn{2}{|c|}{7} & \multicolumn{2}{|c|}{8} \\
\hline & & & \multicolumn{2}{|c|}{$\begin{array}{l}\text { La gerencia ejecuta los } \\
\text { propósitos de la empresa } \\
\text { que la diferencian de las } \\
\text { demás. }\end{array}$} & \multicolumn{2}{|c|}{$\begin{array}{l}\text { Visualizar el futuro } \\
\text { permite a la empresa } \\
\text { motivar a los empleados. }\end{array}$} \\
\hline Alternativas & $\mathbf{F a}$ & Fr \% & $\mathbf{F a}$ & Fr \% & $\mathbf{F a}$ & Fr \% \\
\hline Siempre (S) & 5 & 50 & 3 & 30 & 7 & 70 \\
\hline Casi Siempre (CS) & 1 & 10 & 4 & 40 & 1 & 10 \\
\hline Algunas Veces (AV) & 3 & 30 & 3 & 30 & 2 & 20 \\
\hline Casi Nunca (CN) & 1 & 10 & 0 & 0 & 0 & 0 \\
\hline Nunca (N) & 0 & 0 & 0 & 0 & 0 & 0 \\
\hline Total & 10 & 100 & 10 & 100 & 10 & 100 \\
\hline Media/Ítems & \multicolumn{2}{|c|}{4,00} & \multicolumn{2}{|c|}{4,00} & \multicolumn{2}{|c|}{4,50} \\
\hline Categoría & \multicolumn{2}{|c|}{ Alta aplicación } & \multicolumn{2}{|c|}{ Alta aplicación } & \multicolumn{2}{|c|}{ Muy alta aplicación } \\
\hline Media/indicador & \multicolumn{6}{|c|}{4,17} \\
\hline Categoría & \multicolumn{6}{|c|}{ Alta aplicación } \\
\hline
\end{tabular}

Ahora bien, para el ítem 7 los resultados correspondientes señalan frecuencias de 30\% y $40 \%$ para las alternativas siempre y casi siempre, indicando una tendencia neutral, lo que revela que la gerencia ejecuta los propósitos de la empresa diferenciándola de las demás. Asimismo, la media para este ítem fue de 4, situándose en la categoría alta aplicación.

En cuanto al ítem 8 , se obtuvo frecuencias de $70 \%$ y $10 \%$ para las alternativas siempre y casi siempre, con tendencia positiva, afirmando los encuestados que visualizar el futuro permite a la empresa motivar a sus empleados. Adicionalmente, su media se posicionó en 4,50 ubicándola en la categoría muy alta aplicación en referencia al baremo de interpretación.

Con base a lo anterior, se observa un promedio de 4,17 que indica una alta aplicación de acuerdo a las respuestas dadas por los encuestados, reflejando que dentro de las empresas estudiadas la visión concuerda con lo planteado por Serna (2008) y Chiavenato (2011), quienes indican que la visión provee un marco de referencia sobre lo que una empresa es y quiere ser en el futuro, generando un propósito para la organización en la cual todos los empleados se levantan día tras día, dedicando su tiempo al éxito de la organización.

Los resultados alcanzados para el indicador a su vez ratifican que, la visión como una fuente de inspiración de la cual se extraen fuerzas en los momentos difíciles y sirve de guía para todos los que se comprometen con el negocio hacia una misma dirección. Es decir que, en el direccionamiento estratégico, la visión son los pasos o apuestas para creer indefectiblemente en la realidad del cumplimiento de las metas u objetivos organizacionales, más allá de las circunstancias cuyo rango de afectación esté en el presente. 
En este recorrido se presentan en la tabla 4, los resultados para el indicador objetivos, observándose para el ítem 9 frecuencias de 40\% y 50\% agrupadas en las opciones siempre y casi siempre, señalando una tendencia positiva, indicando que las metas trazadas por la empresa siempre son definidas dentro de los objetivos de la misma. Reflejando a su vez, una media de 4,30 perteneciente a la categoría muy alta aplicación dentro del baremo de interpretación.

Seguidamente, para el ítem 10 se evidencian frecuencias de $40 \%$ y $40 \%$ para las alternativas siempre y casi siempre, con tendencia positiva, afirmando los encuestados que la gerencia asegura que los empleados conozcan los propósitos de la organización, Adicionalmente, para este ítem la media fue de 4,20 ubicándose en la categoría alta aplicación.

De igual forma, para el ítem 11 se observan frecuencias de $40 \%$ y $20 \%$ pertenecientes a las opciones siempre y casi siempre, con tendencia neutral, planteando que los objetivos orientan a los empleados hacia una meta común. En lo que respecta a la media, esta fue de 4 situándose en la categoría alta aplicación.

En este contexto, se observa un promedio de 4,17 señalando una alta aplicación de acuerdo a lo señalado por los encuestados, manifestando que las empresas de fluidos de perforación ubicadas en la división Sur del Lago Trujillo se apoyan en lo expuesto por David (2013), para quien los objetivos de una empresa se centran en la coordinación y establecen una base para las actividades de planeación, organización, dirección y control.

Tabla 4. Objetivos.

\begin{tabular}{|c|c|c|c|}
\hline Indicador & & Principios corporativos & \\
\hline \multirow[t]{2}{*}{ Ítem } & 9 & 10 & 11 \\
\hline & $\begin{array}{l}\text { Las metas trazadas por la } \\
\text { empresa son definidas } \\
\text { dentro de los objetivos de } \\
\text { la misma. }\end{array}$ & $\begin{array}{l}\text { La gerencia asegura que } \\
\text { todos los integrantes de la } \\
\text { organización conozcan los } \\
\text { propósitos de esta. }\end{array}$ & $\begin{array}{l}\text { Los objetivos orientan a } \\
\text { todos los empleados en la } \\
\text { realización de una meta } \\
\text { en común. }\end{array}$ \\
\hline
\end{tabular}

\begin{tabular}{|c|c|c|c|c|c|c|}
\hline Alternativas & $\mathbf{F a}$ & Fr \% & $\mathbf{F a}$ & Fr \% & $\mathbf{F a}$ & $\mathrm{Fr} \%$ \\
\hline Siempre (S) & 4 & 40 & 4 & 40 & 4 & 40 \\
\hline Casi Siempre (CS) & 5 & 50 & 4 & 40 & 2 & 20 \\
\hline Algunas Veces (AV) & 1 & 10 & 2 & 20 & 4 & 40 \\
\hline Casi Nunca (CN) & 0 & 0 & 0 & 0 & 0 & 0 \\
\hline Nunca (N) & 0 & 0 & 0 & 0 & 0 & 0 \\
\hline Total & 10 & 100 & 10 & 100 & 10 & 100 \\
\hline Media/Ítems & \multicolumn{2}{|c|}{4,30} & \multicolumn{2}{|c|}{4,20} & \multicolumn{2}{|c|}{4,00} \\
\hline Categoría & \multicolumn{2}{|c|}{ Muy alta aplicación } & \multicolumn{2}{|c|}{ Alta aplicación } & \multicolumn{2}{|c|}{ Alta aplicación } \\
\hline Media/indicador & \multicolumn{6}{|c|}{4,17} \\
\hline Categoría & \multicolumn{6}{|c|}{ Alta aplicación } \\
\hline
\end{tabular}


Los resultados alcanzados por el indicador, ratifican que, los objetivos aluden a propósitos esenciales de la empresa orientando al equipo humano hacia las metas trazadas. De manera que, las empresas bajo estudio deben realizar, como mínimo, una revisión anual del estado de avance y cumplimiento de los objetivos y metas propuestos tanto en el corto como en el largo plazo, de esta manera contará con información que le indicará si sus esfuerzos están o no siendo efectivos, y pueda tomar decisiones oportunamente e implementar los correctivos necesarios para lograr con éxito sus metas, tal como se estable en el direccionamiento estratégico.

Con relación al indicador políticas, en la tabla 5, se observa para el ítem 12 frecuencias de $40 \%$ y $20 \%$ para las alternativas siempre y casi siempre, con tendencia neutral, afirmando los encuestados que para la toma de decisiones la alta gerencia toma en consideración las políticas de la empresa. Al mismo tiempo le acompaña una media de 3,80 colocándose en la categoría alta aplicación en referencia al baremo de interpretación.

Tabla 5. Políticas.

\begin{tabular}{|c|c|c|c|c|c|c|c|c|}
\hline \multirow{3}{*}{$\begin{array}{c}\text { Indicador } \\
\text { Ítem }\end{array}$} & \multicolumn{8}{|c|}{ Políticas } \\
\hline & \multicolumn{2}{|c|}{12} & \multicolumn{2}{|c|}{13} & \multicolumn{2}{|c|}{14} & \multicolumn{2}{|c|}{15} \\
\hline & \multicolumn{2}{|c|}{$\begin{array}{l}\text { Para la toma de } \\
\text { decisiones la alta } \\
\text { gerencia toma en } \\
\text { cuenta las políticas } \\
\text { de la organización. }\end{array}$} & \multicolumn{2}{|c|}{$\begin{array}{l}\text { Durante el } \\
\text { desarrollo de sus } \\
\text { funciones los } \\
\text { empleados toman } \\
\text { en consideración } \\
\text { las políticas de la } \\
\text { empresa. }\end{array}$} & \multicolumn{2}{|c|}{$\begin{array}{l}\text { Las acciones se } \\
\text { encuentran } \\
\text { delimitadas en base } \\
\text { a un grupo de reglas } \\
\text { que rigen la } \\
\text { organización. }\end{array}$} & \multicolumn{2}{|c|}{$\begin{array}{l}\text { Las políticas } \\
\text { corporativas son } \\
\text { entendidas por todos } \\
\text { los miembros de la } \\
\text { organización }\end{array}$} \\
\hline Alternativas & $\mathbf{F a}$ & Fr \% & $\mathbf{F a}$ & Fr \% & $\mathrm{Fa}$ & Fr \% & $\mathrm{Fa}$ & Fr \% \\
\hline Siempre (S) & 4 & 40 & 3 & 30 & 3 & 30 & 2 & 20 \\
\hline Casi Siempre (CS) & 2 & 20 & 1 & 10 & 2 & 20 & 6 & 60 \\
\hline Algunas Veces (AV) & 2 & 20 & 4 & 40 & 5 & 50 & 2 & 20 \\
\hline Casi Nunca (CN) & 2 & 20 & 2 & 20 & 0 & 0 & 0 & 0 \\
\hline Nunca (N) & 0 & 0 & 0 & 0 & 0 & 0 & 0 & 0 \\
\hline Total & 10 & 100 & 10 & 100 & 10 & 100 & 10 & 100 \\
\hline Media/Ítems & \multicolumn{2}{|c|}{3,80} & \multicolumn{2}{|c|}{3,50} & \multicolumn{2}{|c|}{3,80} & \multicolumn{2}{|c|}{4,00} \\
\hline Categoría & \multicolumn{2}{|c|}{ Alta aplicación } & \multicolumn{2}{|c|}{ Alta aplicación } & \multicolumn{2}{|c|}{ Alta aplicación } & \multicolumn{2}{|c|}{ Alta aplicación } \\
\hline Media/indicador & \multicolumn{8}{|c|}{3,78} \\
\hline Categoría & \multicolumn{8}{|c|}{ Alta aplicación } \\
\hline
\end{tabular}

Respecto al ítem 13, se visualizan frecuencias de 30\% y $10 \%$ agrupadas en las opciones siempre y casi siempre, indicando tendencia negativa, lo que demuestra que la mayoría de los encuestados concuerda que no es muy frecuente que las políticas de la empresa sean tomadas en consideración para el desarrollo de las funciones por parte de los empleados de la misma, Adicionalmente la media para este ítem fue de 3,50 situándose en la categoría alta aplicación. 
En referencia al ítem 14 se obtuvo frecuencias de 30\% y 20\% para las alternativas siempre y casi siempre, con tendencia neutral, lo que revela que solo en algunos casos las acciones son limitadas en base a las reglas que rigen a la organización. Igualmente, la media fue de 3,80 ubicándose en la categoría alta aplicación.

Ahora bien, para el ítem 15 se presentan frecuencias de 20\% y 60\% agrupadas en las opciones siempre y casi siempre, con tendencia positiva, acotando que la mayoría de los encuestados opina que las políticas son entendidas por todos los miembros de la empresa. Asimismo, la media para este ítem fue de 4, situándose en la categoría alta aplicación.

En este sentido, se observa un promedio de 3,78 mostrando alta aplicación según las respuestas dadas por los encuestados, poniendo en evidencia que dentro de las empresas bajo estudio, los resultados referida a las políticas coinciden con lo expuesto por Melinkoff (2005), quien afirma que las mismas constituyen una norma de acción y a su vez son un conjunto de reglas y orientaciones que delimitan la acción administrativa.

Con estos resultados alcanzados para el indicador, se corrobora que, las políticas constituyen los lineamientos a seguir en las organizaciones que sirven como una guía para las acciones desempeñadas por los trabajadores en sus actividades laborales. Así las cosas, las políticas deben incluir las directrices, reglas y procedimientos con el propósito de propiciar esfuerzos encaminados al logro de los objetivos establecidos en el direccionamiento estratégica.

A propósito de lo mostrado en la tabla 6 referido al indicador valores, se muestran frecuencias de $40 \%$ y $40 \%$ para las alternativas siempre y casi siempre, con tendencia positiva, acotando los encuestados que los valores de la empresa son conocidos por los miembros de la misma; además, se obtuvo una media de 4,10 la cual se ubicó en la categoría de alta aplicación dentro del baremo.

Por su parte, para el ítem 17 se evidencian frecuencias de 20\% y 50\% agrupadas en las opciones siempre y casi siempre, con tendencia neutral, afirmando que en la mayoría de los casos los empleados orientan su trabajo en base a los valores de la organización. Adicionalmente, se obtuvo una media de 3,90 que coincide con la categoría alta aplicación del baremo. 
Tabla 6. Valores.

\begin{tabular}{|c|c|c|c|c|c|c|}
\hline \multirow{3}{*}{$\begin{array}{c}\text { Indicador } \\
\text { Ítem }\end{array}$} & \multicolumn{6}{|c|}{ Principios corporativos } \\
\hline & \multicolumn{2}{|c|}{16} & \multicolumn{2}{|c|}{17} & \multicolumn{2}{|c|}{18} \\
\hline & \multicolumn{2}{|c|}{$\begin{array}{l}\text { Los valores de la } \\
\text { organización son } \\
\text { conocidos por todos los } \\
\text { miembros de la misma. }\end{array}$} & \multicolumn{2}{|c|}{$\begin{array}{l}\text { Los empleados orientan } \\
\text { su trabajo en base a los } \\
\text { valores de la organización. }\end{array}$} & \multicolumn{2}{|c|}{$\begin{array}{l}\text { El comportamiento de los } \\
\text { empleados se encuentra } \\
\text { condicionado por los } \\
\text { valores corporativos. }\end{array}$} \\
\hline Alternativas & $\mathbf{F a}$ & Fr \% & $\mathbf{F a}$ & Fr \% & $\mathbf{F a}$ & Fr \% \\
\hline Siempre (S) & 4 & 40 & 2 & 20 & 0 & 0 \\
\hline Casi Siempre (CS) & 4 & 40 & 5 & 50 & 7 & 70 \\
\hline Algunas Veces (AV) & 1 & 10 & 3 & 30 & 2 & 20 \\
\hline Casi Nunca (CN) & 1 & 10 & 0 & 0 & 1 & 10 \\
\hline Nunca $(N)$ & 0 & 0 & 0 & 0 & 0 & 0 \\
\hline Total & 10 & 100 & 10 & 100 & 10 & 100 \\
\hline Media/Ítems & \multicolumn{2}{|c|}{4,10} & \multicolumn{2}{|c|}{3,90} & \multicolumn{2}{|c|}{3,60} \\
\hline Categoría & \multicolumn{2}{|c|}{ Alta aplicación } & \multicolumn{2}{|c|}{ Alta aplicación } & \multicolumn{2}{|c|}{ Alta aplicación } \\
\hline Media/indicador & \multicolumn{6}{|c|}{3,87} \\
\hline Categoría & \multicolumn{6}{|c|}{ Alta aplicación } \\
\hline
\end{tabular}

De igual forma, para el ítem 18 se observa frecuencias de $0 \%$ y 70\% para las opciones siempre y casi siempre, con tendencia neutral, donde la mayoría de los sujetos encuestados concuerdan que frecuentemente el comportamiento de los empleados se encuentra condicionado por los valores corporativos. Asimismo, la media para este ítem fue de 3,60, situándose en la categoría alta aplicación.

Ahora bien, se obtuvo un promedio de 3,87, haciendo notar una alta aplicación de acuerdo a lo señalado por los encuestados, evidenciando que para las empresas objeto de estudio los valores concuerdan con lo expuesto Serna (2008) y Aceves (2007), quienes afirman que los valores son de relevancia para cada una de las personas que conforman una organización, siendo estos principios que norman las conductas y ciertos comportamientos específicos y que se muestran con orgullo y se defienden.

Dichos resultados para el indicador, confirman que, los valores son de relevancia para cada una de las personas que conforman la organización, quienes deben saber que su ejecución traerá consigo beneficios tanto para ellos como para la empresa. En efecto, los valores son el ancla para permanecer incólume en lo que una empresa considera que es correcto y loable en la conjunción de sus intereses corporativos y el bienestar social, siendo los estándares de calidad que giran en torno a una fuerte de convicción de lo deseable al manejar sabiamente cualquier dificultad que desvía su enfoque de negocios y los esfuerzos de la hoja de ruta trazada del direccionamiento estratégico, no cometiendo el error de tomar "atajos" hacia el éxito. 
En referencia a la tabla 7, considerando los resultados obtenidos para cada uno de los indicadores pertenecientes al direccionamiento estratégico, se obtuvo un promedio de 4,01 señalando una alta aplicación del mismo dentro de las empresas de fluidos de perforación ubicadas en la división Sur del Lago Trujillo, las cuales se apoyan en lo expuesto por Amaya (2005), quien afirma que las organizaciones para crecer, generar utilidades y permanecer en el mercado deben tener muy claro hacia dónde van, es decir, haber definido su direccionamiento estratégico.

Tabla 7. Direccionamiento estratégico.

\begin{tabular}{ccc}
\hline Indicador & Media & Categoría \\
\hline Principios corporativos & 4,40 & Muy alta aplicación \\
\hline Misión & 3,65 & Alta aplicación \\
\hline Visión & 4,17 & Alta aplicación \\
\hline Objetivos & 4,17 & Alta aplicación \\
\hline Políticas & 3,78 & Alta aplicación \\
\hline Valores & 3,87 & Alta aplicación \\
\hline Total & $\mathbf{4 , 0 1}$ & Alta aplicación \\
\hline
\end{tabular}

Sobre la base de los valores resultantes para dicha dimensión, se ratifica que, el direccionamiento estratégico es un enfoque sistemático hacia una responsabilidad mayor y cada vez más importante de la alta dirección. Indicando asimismo que, el direccionamiento estratégico influye en los planes operativos que debe tener el desarrollo exhaustivo del qué, cómo, cuándo y quién, dejando claro los hitos de cumplimiento de la estrategia y los factores críticos de éxito para fomentar la responsabilidad en el proceso y tomar decisiones claves para no paralizar cualquier actividad en ejecución.

\section{CONCLUSIONES}

$\mathrm{S}$ e pudo determinar que existe una alta aplicación del direccionamiento estratégico, por lo que a través de él se formulan los propósitos de las empresas de Fluido de Perforación, donde se consignan los objetivos definidos para un largo plazo que apuntan a la perdurabilidad, sostenibilidad y crecimiento de las mismas y que sirve de marco referencial para los objetivos y lineamientos consagrados en su plan estratégico.

Así las cosas, el direccionamiento estratégico permite la toma de decisiones del gerente, las cuales deben asumirse como transformaciones, que conduzcan a las organizaciones por nuevos rumbos, nuevos procesos, nuevos objetivos, nuevas estrategias, nuevas tecnologías e incluso nuevos riesgos; y a nuevos comportamientos y actitudes del capital humano. Todo ello, utilizando herramientas que permitan un desarrollo gerencial dirigido e incrementar la habilidad del liderazgo y resaltar los valores institucionales. 


\section{REFERENCIAS}

Aceves, V. (2007). Dirección estratégica. Primera edición. Editorial Mc. Graw Hill. México

Amaya, J. (2005). Gerencia: planeación y estrategia. Universidad Santo Tomás. Colombia

Chiavenato, I. (2011). Planificación estratégica: fundamentos y aplicaciones. Segunda edición. Mc. Graw Hill. Colombia

David, F. (2013). Conceptos de administración estratégica. Pearson Educación. México

Dess, G.; y Lumpkin, G. (2003). Dirección estratégica. Madrid: mc Graw Hill Interamericana

Hernández, S.; Fernández C., y Baptista, L. (2010). Metodología de la investigación. Editorial Mc Graw Hill. México.
Huisa, E. (2007). Planeamiento estratégico, slideshare.net. Disponible en: http://es.slideshare.net/elizabethuisa/planeamie ntoestratgico

Melinkoff, R. (2005). Los procesos administrativos. Editorial Panapo, C.A. Venezuela

Rojas, M. y Medina, L. (2012). Planeación estratégica. Bogotá: Ediciones de la U.

Serna, H. (2008). Gerencia estratégica. Décima edición. Editorial 3r Editores. Bogotá, Colombia 